\section{The Estimate of Relative Increase in Stroke Volume of a Heart under Influence of Low Frequency Mechanical Vibrations}

\author{
Nahirniak V. M. ${ }^{1 * 0}$, Tsyhykalo O. V.2, Oliinyk I. ${ }^{3}$, Pentelej- \\ chuk N. ${ }^{4}$, Stoliar D. ${ }^{4}$, Lavriv L. ${ }^{5}$, Andrushchak L. ${ }^{6}$
}

\begin{abstract}
Background: An automatic massage produces health improving effects. After a single automatic massage, patients admit a feeling of invigoration, and a sense of relaxation. Some quantitative characteristics of physical effects produced by the automatic massage on the body, including the work of a heart were unavailable at that moment.

Objective: This study aims to find a quantitative impact of periodic low frequency mechanical vibrations on the relative change in a heart stroke volume.

Material and Methods: In this experimental study, the patients were exposed to the low frequency $(12 \mathrm{~Hz})$ planar mechanical vibrations. The blood pressures were measured before and after the automatic massage. Based on the measured values in the arterial blood pressure, a relative change in a stroke volume (SV) in patients was calculated.
\end{abstract}

Results: The increased systolic blood pressure was $6.3 \pm 2.0 \mathrm{~mm} \mathrm{Hg}$ in women and $11.1 \pm 2.7 \mathrm{~mm} \mathrm{Hg}$ in men. The increased diastolic pressure was $1.9 \pm 1.2 \mathrm{~mm} \mathrm{Hg}$ in women, and $4.9 \pm 1.5 \mathrm{~mm} \mathrm{Hg}$ in men. The minor increase in heart rate for women was $1.2 \pm 1.0$ beats per minute, and $1.2 \pm 2.0$ beats per minute for men. The assessment of stroke volume changes provided $9.6 \%$ and $7.1 \%$ increase during systole and diastole in male patients, respectively, and corresponding $6.7 \%$ and $4.7 \%$ increases for female patients.

Conclusion: The results of our work confirmed an increase in the systolic and diastolic blood pressures under the influence of periodic mechanical vibration of low frequency. We believe that the registered increase in blood pressure is a proof of the increase in a stroke volume (SV).

Citation: Nahirniak VM, Tsyhykalo OV, Oliinyk I, Pentelejchuk N, Stoliar D, Lavriv L, Andrushchak L. The Estimate of Relative Increase in Stroke Volume of a Heart under Influence of Low Frequency Mechanical Vibrations. J Biomed Phys Eng. 2021;11(1):85-92. doi: 10.31661/jbpe.v0i0.2003-1093.

\section{Keywords}

Stroke Volume; Cardiac Output; Arterial Blood Pressure; Whole Body Vibrations; Hypertension

\section{Introduction}

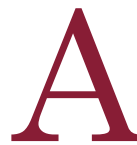
$\mathrm{n}$ automatic massage lasting at least 10 min produces health improving effects in patients [1-6]. Even after a single automatic massage, patients admit a feeling of invigoration and energy, vitality, reduced fatigue, and a body-wide sense of relaxation. After a se-
${ }^{1} \mathrm{PhD}$, Department of Biophysics and Medical Informatics, Bukovinian State Medical University, Chernivtsi, Ukraine ${ }^{2} \mathrm{MD}$, Department of Histology, Cytology and Embryology, Bukovinian State Medical University, Chernivtsi, Ukraine

${ }^{3} \mathrm{MD}$, Department of

Pathological Anatomy, Bukovinian State Medical University, Chernivtsi, Ukraine

${ }^{4} \mathrm{PhD}$, Department of Histology, Cytology and

Embryology, Bukovinian

State Medical University,

Chernivtsi, Ukraine

${ }^{5} \mathrm{MD}$, Department

of Anatomy, Clinical

Anatomy and Operative

Surgery, Bukovinian

State Medical University,

Chernivtsi, Ukraine

${ }^{6} \mathrm{PhD}$ Candidate, Depart-

ment of Histology, Cytol-

ogy and Embryology,

Bukovinian State Medi-

cal University, Chernivtsi,

Ukraine

*Corresponding author:

V. M. Nahirniak

Department of Bio-

physics and Medical

Informatics, Bukovinian

State Medical University,

Chernivtsi, Ukraine

E-mail: volnag@gmail.

com

Received: 31 March 2020 Accepted: 6 June 2020 
ries of massages, they admit general improvement of health, increased physical activity, and reduction of chronic pains in joints.

One of the factors which may contribute to repair of injured tissues is blood supply to the affected areas. Previous studies have shown the effect of periodic mechanical oscillations of low frequency $(20-60 \mathrm{~Hz})$ on peripheral blood flow (14\% increase), diastolic pressure, carboxyhemoglobin levels, and transverse size of human blood vessels $[1,2]$.

Some quantitative characteristics of physical effects produced by the automatic massage on the body, including the work of a heart were unavailable at that moment [3]. Our goal in this work was to find a quantitative impact of periodic low frequency mechanical vibrations (720 oscillations per min) on the relative change in a heart stroke volume in men and women.

Traditional manual therapeutic massage may be prescribed in many cases due to its wellknown healing ability. According to the traditional views, the healing and invigorating effects of the massage are attributed mainly to the improved blood and lymph circulation [4, 5]. Manual massage produces physiological changes in a body [6]. The recent studies demonstrated that it produces reduction of systolic $(10 \mathrm{mmHg})$, diastolic $(5.8 \mathrm{mmHg})$ pressures, and in the heart rate (by 10.8 beats per min), respectively.

The use of automatic massagers has some advantages over manual massages. First, it's because of their affordability and accessibility. Second, a user may control the effect produced by the massaging machine and repeat the same massaging session later.

The growth of the blood flow and the increase in diastolic pressure in vascular system were observed in people exposed to mechanical vibrations. The authors studied the influence of low-frequency vibrations $(60 \mathrm{~Hz})$ on the peripheral blood circulation in humans. The authors noted that the increase in blood circulation is not only associated with meta- bolic requirements of tissues [1].

Based on the obtained results on the increase of arterial blood pressure in patients whose low extremities were exposed to the low frequency $(12 \mathrm{~Hz})$ mechanical vibrations for 10 min, the authors made conclusions about the increase of the effective radius of the blood vessels in low extremities [7].

Well-developed muscles and vasculature and unrestricted blood circulation promote faster recovery of body tissues. Physical exercises can increase a size of capillaries by 100 times. Rhythmic muscle contractions accelerate the peripheral blood circulation. Studies have shown that low-frequency $(30 \mathrm{~Hz})$ vibrations of the whole body for 9 min increase the blood flow and the volume of blood in the muscles of the lower extremities [8].

It was confirmed that a use of low-frequency mechanical vibrations helps in the elimination of chronic pain in a lower part of spine [9]. The increase of a heart rate and a blood pressure due to periodic mechanical vibrations was recorded in this work.

In the other work, the increased blood pressure and heart rate by an average of 10 percent was recorded due to the low frequency $(15 \mathrm{~Hz})$ vibration transfer to the chest via hand which lasted approximately $3 \mathrm{~min}$ [10].

There was no significant change in blood pressure and heart rate in the group of people after their exposure to a 5-min session of mechanical vibrations with frequency of $40 \mathrm{~Hz}$ and amplitude in $1.9 \mathrm{~mm}$ [11]. However, these measurements were conducted after the 5-min break, in contrary to our studies, where the measurements were conducted immediately after the automatic massage. The increased blood flow, which the authors observed, was attributed to the physiological changes in the peripheral circulatory system.

It is noted the positive effect of mechanical vibrations on the diameter of the arteries and a lack of impact of vibrations on a body's temperature [12].

Exposure of feet to the low frequency (30 
Stroke Volume Increase Due to a Vibrational Massage

$\mathrm{Hz}$ ) vibrations demonstrated a short-term increase in skin blood flow [13].

Whole body vibrations at low frequencies $(10-30 \mathrm{~Hz})$ are an effective method to increase a leg's blood flow and to activate a muscle mass in patients [14]. They attributed the increased blood flow to the myogenic response in vasculature.

\section{Material and Methods}

In this experimental study, planar circular mechanical vibrations were generated by the industrial Vibro STSEK S780 model manufactured by Tianshi (Tianjin, China). A photograph of the apparatus is shown in Figure 1.

Using a mechanical vibratory massage at the wellness centers confirms the generall action of this apparatus. In practice, it is used in case of health problems with the musculoskeletal system, arthritis, varicose veins, chronic fatigue, insomnia, joints pain, and digestive disorders. Duration of vibration massage is controlled by a built-in timer. The central vibrating platform generates vibrations in two planes with amplitude of approximately $6 \mathrm{~mm}$. The frequency of vibrations is fixed and equal to 720 vibrations per min.

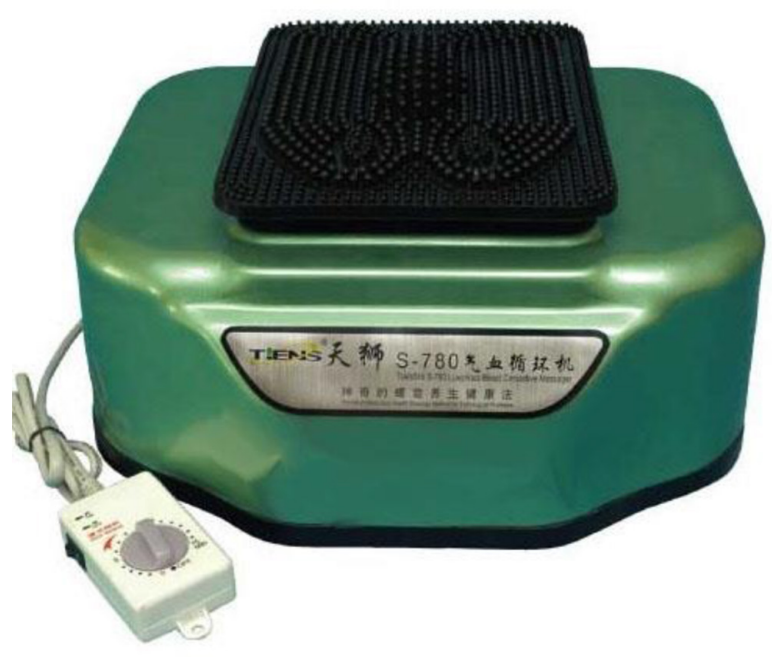

Figure 1: The apparatus for periodic mechanical vibrations with a timer and a rubber platform.
There were a few different positions which patients may take on the platform. In our case, the patients were sitting on the rubber platform of automatic massager with the fully stretched legs as shown in Figure 2.

In this position, mainly thoracic part of a body is affected by the vibrations. The duration of vibratory massage in all experiments was ten min.

The personal blood pressure monitor Rossmax MS60 (Taipei, Taiwan) was used for pressure measurements. The arm cuffs were positioned approximately at $2 \mathrm{~cm}$ above the left hand elbow. In order to increase the accuracy of measurements of blood pressure before vibratory massage session, the pressure was measured twice with interval of $2 \mathrm{~min}$. The last was necessary to restore blood circulation in hand before the first measurement. After two consecutive measurements, the obtained results were averaged. Patients had rested on

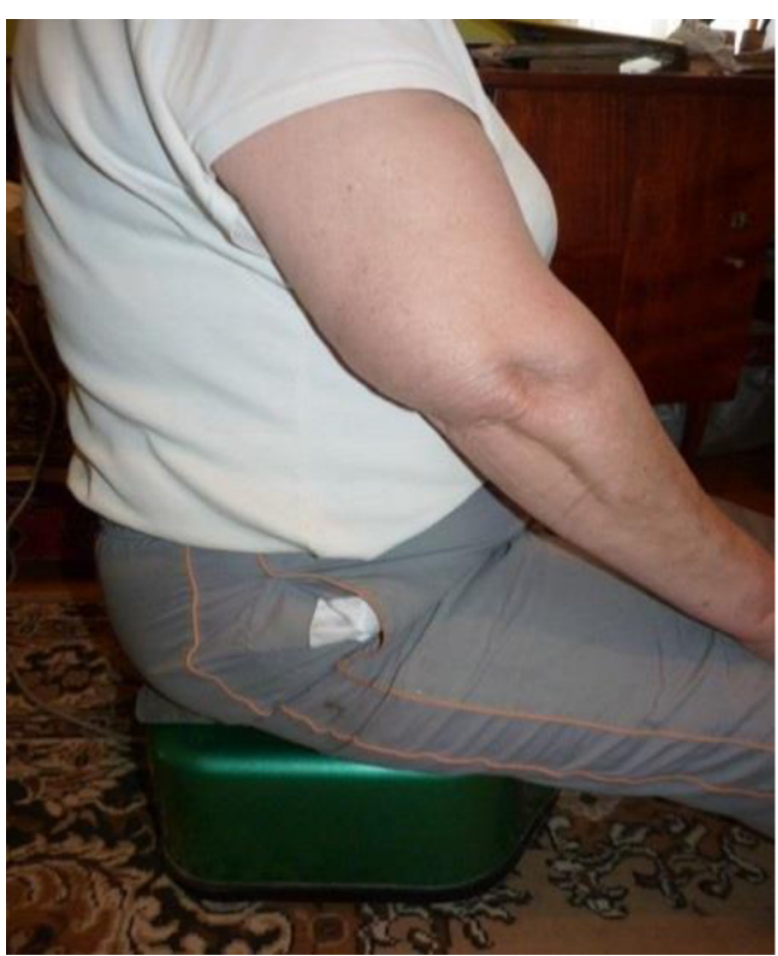

Figure 2: A patient sitting on the rubber platform of the automatic massager. 
a chair for $5 \mathrm{~min}$ before the first pressure and heart rate (HR) measurements were conducted. The measurement of blood pressure and heart rate was repeated right after the completion of vibratory massage. It was noted that, blood pressure and heart rate return to their previous normal values within $5 \mathrm{~min}$ after the massage. This measurement protocol was chosen after preliminary studies. Processing of results was conducted in MS Excel 2010 (Redmond, WA, USA). In our experiments, at first, we had carried out the measurements of their quantitative impact on blood pressure and a heart rate in men and women of different age groups and previous medical history, including people with hypertension and hypotension. Then, based on the obtained results and using the Hagen-Poiseuille model for a blood circulatory system, we made calculations for a relative change in a heart stroke volume in humans.

\section{Participants}

The target group included 16 men and 14 women aged from 20 to 60 years. Participants included the hypotensive, hypertensive pa- tients and patients with normal blood pressure. Most of them denied a regular use of medications that regulate blood pressure. Their weight ranged from 70 to $126 \mathrm{~kg}$.

\section{Results}

Both groups of participants admitted the improved state of health after a $10 \mathrm{~min}$ automatic massage of the chest portion of a body.

The experimental results are shown in the diagram in Figure 3. The increased systolic blood pressure was $6.3 \pm 2.0 \mathrm{mmHg}$ in women and $11.1 \pm 2.7 \mathrm{~mm} \mathrm{Hg}$ in men. The increased diastolic pressure was $1.9 \pm 1.2 \mathrm{~mm} \mathrm{Hg}$ in women and $4.9 \pm 1.5 \mathrm{~mm} \mathrm{Hg}$ in men. The average results based on the increase in systolic and diastolic blood pressures are presented along with the standard errors. Also, we have noticed a minor increase in a heart rate. The increase in heart rate for women was $1.2 \pm 1.0$ beats per min and $1.2 \pm 2.0$ beats per min for men.

The observed increase in the men and women with systolic and diastolic arterial blood pressure is summarized in Table 1.

According to the Hagen-Poiseuille equa-

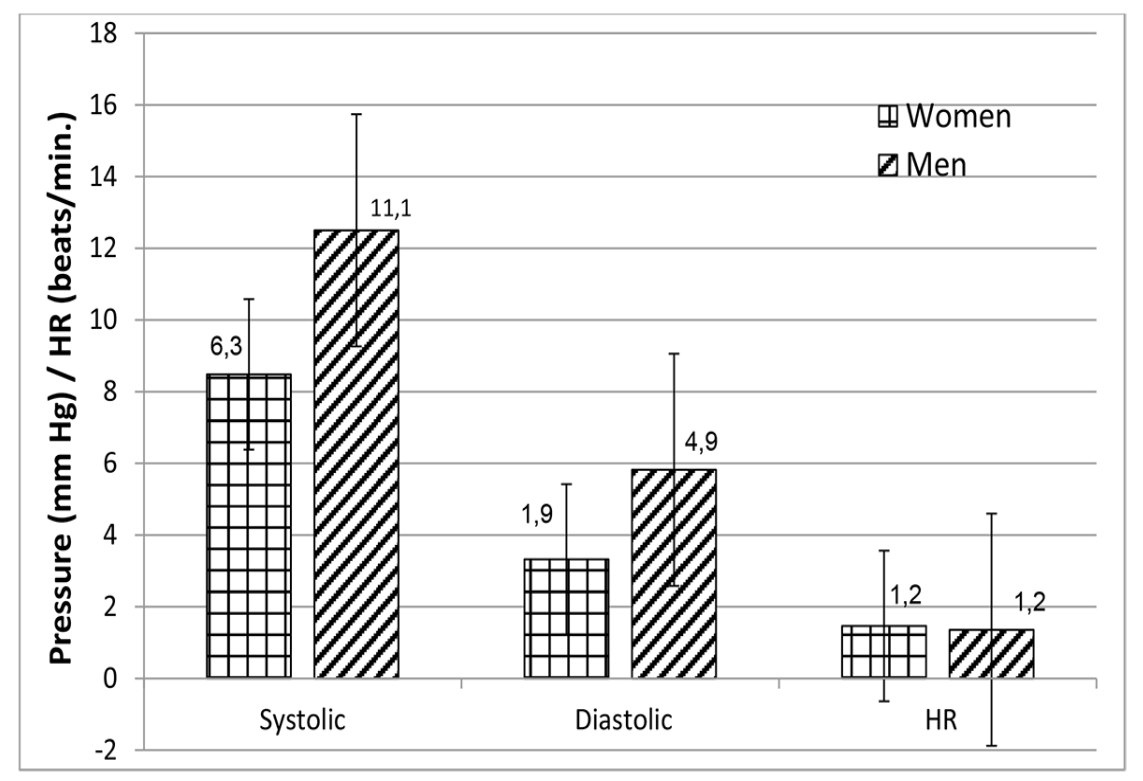

Figure 3: The experimental results on the increases in arterial blood pressure and heart rate. Results are shown along with the standard errors. 
Table 1: The observed values of the increased systolic and diastolic pressures and the heart rates in men and women after a whole body automatic massage. The data are presented along with standard errors.

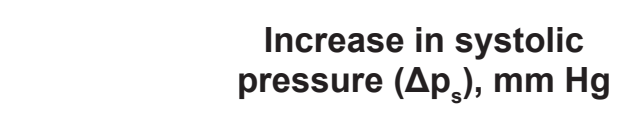

Increase in diastolic pressure Change in heart rate $\left(\Delta p_{\mathrm{d}}\right), \mathrm{mm} \mathrm{Hg}$ (HR), beats/min

\begin{tabular}{cccc}
\hline Men & $11.1 \pm 2.7$ & $4.9 \pm 1.5$ & $1.2 \pm 2.0$ \\
\hline Women & $6.3 \pm 2.0$ & $1.9 \pm 1.2$ & $1.2 \pm 1.0$
\end{tabular}

tion, the systolic and diastolic blood pressure is given by the following formula:

$$
p=\frac{8 \eta L C O}{\pi R^{4}}=\frac{8 \eta L S V H R}{\pi R^{4}}
$$

Where $\eta$ is viscosity of blood, $R$ is the effective radius of vascular system, and $L$ is its effective length. There are a few factors which may result in the observed blood pressure change in our conditions, including a stroke volume, a heart rate, and an effective radius blood vessels in thoracic and abdominal parts of the patient's body. If the heart rate (HR) and the radius remain unchanged during the experiment, only the change in stroke volume (SV) defines the change in the blood pressure (p):

$$
d p=\frac{8 \eta L H R}{\pi R^{4}} \cdot d S V
$$

The results of our experiments showed no substantial changes in a heart rate. We also assume that this frequency vibratory motion does not affect the size of blood vessels. This assumption is based on the results of our previous work and we observed the increase of the size of blood vessels under influence of the same automatic vibrational massage [7]. If the same happened in these experiments, it would have led to the decrease in blood pressure which we did not observe. Then, using the last equation, the relative change in a stroke volume may be estimated as follows:

$$
\frac{S V_{2}-S V_{1}}{S V_{1}}=\frac{d S V}{S V} \approx \frac{d p}{p}, H R_{1} \approx H R_{2}
$$

Where $S V_{2}$ and $S V_{1}$ are the stroke volumes after and before the automatic massage, $d p \approx p_{2}-p_{1}$ is the change in blood pressure before $\left(p_{1}\right)$ and after $\left(p_{2}\right)$ massage.

The values for the change in stroke volume were assessed individually for each patient among men and women. The averaged results after these calculations for both groups are shown in the Table 2.

\section{Discussion}

A blood pressure gradient exists across the systemic circulatory system, starting from an aorta and ending in vena cavas. Evaluation of the overall pressure drop in the big circle of blood circulation and pressure drop in a separate section of the human circulatory system can be made graphically. Average systolic and diastolic pressures within the vascular system are shown in Figure 4. The increase in both, systolic and diastolic blood pressure will in-

Table 2: The relative increases in a stroke volume after the action of vibrational massage.

Increase during systolic period $\left(\Delta \mathrm{SV}_{\mathrm{SSV}}\right), \%$ Increase during diastolic period $\left(\Delta \mathrm{SV} / \mathrm{SV}_{1}\right), \%$

\begin{tabular}{ccc}
\hline Men & 9.6 & 7.1 \\
\hline Women & 6.7 & 4.7
\end{tabular}




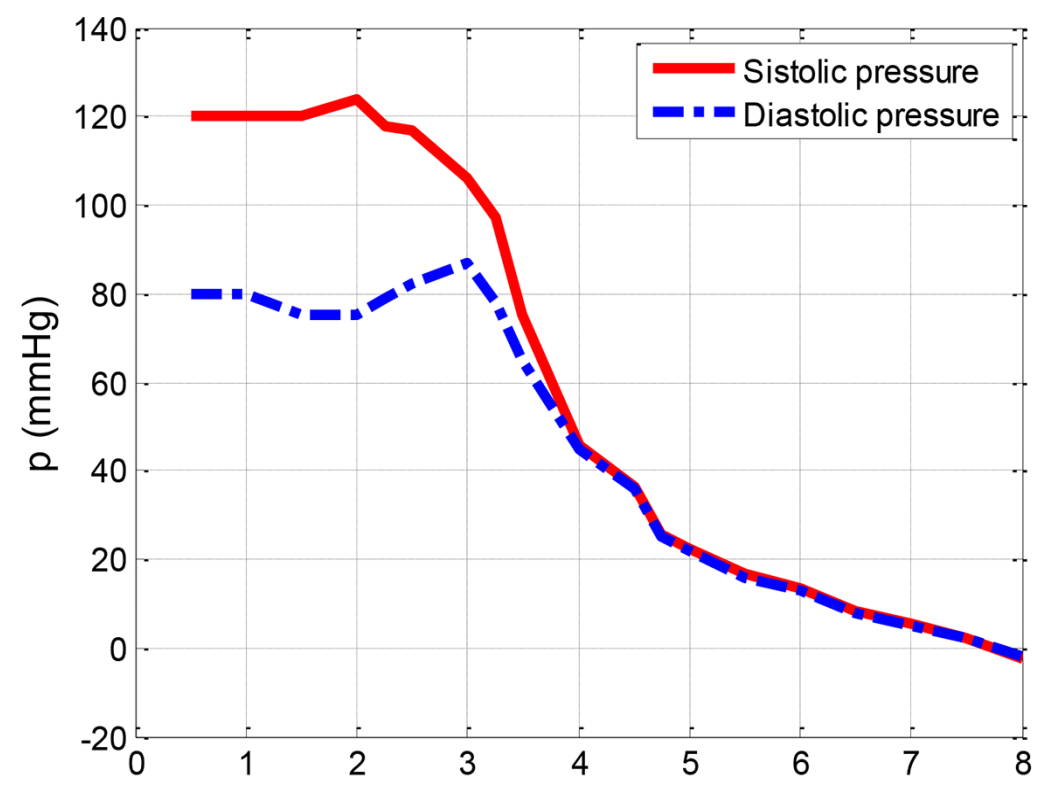

Figure 4: The average normal systolic and diastolic pressures at the different parts of a systemic blood circulatory system of humans. 1- Blood pressure in aorta, 8- blood pressure in vena caves. (Reproduced from Folkow B, Neil E. Circulation. 1971. p. 593. [15])

crease the pressure gradient and, consequently, it will increase the volumetric rate of blood circulating through vessels.

Theoretically, according to the law of Hagen-Poiseuille, an increase of the volume of blood $S V$ in the bloodstream is directly proportional to the blood pressure gradient $\Delta \mathrm{p}[9]$ :

$$
\Delta p=\frac{8 \eta L S V}{\pi R^{4}} . H R
$$

Where $\eta$-dynamic viscosity of blood, $L$ is the effective length of circulatory system, and $R$ - effective average radius of blood vessels. This applies to both systolic and diastolic periods in a cardiac cycle. Our experimentally measured high blood pressure is an indirect justification of this assumption. Also, the increase in total blood flow is directly proportional to the increase in a heart rate.

Reduced blood volume (for example due to excessive sweating) reduces blood pressure in a short term. Long-term homeostatic mechanisms compensate bringing blood volume and blood pressure back up to normal levels. The increased blood volume due to, for example, water retention or excessive salt intake, increases blood pressure in a short term. The action of vibrational massage, which we observed in this study, also increases blood pressure, but in this case, due to the increased cardiac output (CO), and, which leads, in turn, to the increase of the volumetric rate of circulating blood. The increased blood flow provides the healing effect in injured tissues and organs. In both cases, blood pressure comes back to the normal value sometime after their actions.

\section{Conclusion}

The results of our work confirmed an increase in the systolic and diastolic blood pressures under the influence of periodic mechanical vibration of low frequency. We believe that the registered increase in blood pressure is a proof of the increase in a stroke volume (SV) and, consequently, the increase in volumetric blood circulation rate in the human vascular system. From the therapeutic standpoint, this 
process potentially increases the oxygen content in the blood due to increased volume of blood circulation. Automatic vibrational massage demonstrates healing ability. We believe that the automatic vibratory effect of using the apparatus STSEK is achieved in part due to acceleration of circulation of blood, improving its blood circulation and increase its oxygenation. Thus, mechanical vibrations facilitate the circulation of blood in muscle tissues by promoting cell regeneration. Although, its action is limited in time, the prolonged use of the sessions of automatic massage will increase its cumulative non-medicamentous effect of the vibrational massage.

Further investigations may help to clarify the effect of frequency, amplitude and duration of mechanical vibrations on the arterial blood pressure and eventually its therapeutic healing effect. Based on our experience, we think the amplitude of vibrations must be greater than $5 \mathrm{~mm}$ and the duration of automatic massage must be at least 5-10 min to produce the therapeutic effect. The frequency must be greater than $10 \mathrm{~Hz}$.

\section{Acknowledgment}

The authors would like to thank the health center "Tian Shi" in Chernivtsi (UKRAINE) for the opportunity to conduct current research.

\section{Conflict of Interest}

None

\section{References}

1. Button C, Anderson N, Bradford C, et al. The effect of multidirectional mechanical vibration on peripheral circulation of humans. Clin Physiol Funct Imaging. 2007;27(4):211-6. doi: 10.1111/j.1475-097X.2007.00739.x. PubMed PMID: 17564669.

2. Nahirnyak V, Branashko E, Kifichuk IG. Influence of periodic mechanical vibrations on human arterial blood pressure. Bukovinian Medical Herald. 2017;21(81):96-9. doi: 10.24061/2413-0737.XXI.1.81.2017.20.

3. Games KE, Sefton JM, Wilson AE. Whole-
Body Vibration and Blood Flow and Muscle Oxygenation: A Meta-Analysis. Journal of Athletic Training. 2015;50(5):542-9. doi: 10.4085/1062-6050-50.2.09. PubMed PMID: 25974682. PubMed PMCID: PMC4560014.

4. Sense Massage Therapy. The Physiological Effects of Massage on the Body. Available from: https://www.sensemassage.co.uk/blog/75the-physiological-effects-of-massage-on-thebody.

5. Vickers A, Zollman C, Reinish JT. Massage therapies. West J Med. 2001;175(3):202-4. PubMed PMCID: PMC1071543.

6. Kaye AD, Kaye AJ, Swinford J, Baluch A, Bawcom BA, Lambert TJ, Hoover JM. The Effect of Deep-Tissue Massage Therapy on Blood Pressure and Heart Rate. Journ of Alternative and Complem Medicine. 2008;14(2):125-8. doi: 10.1089/acm.2007.0665. PubMed PMID: 18315516.

7. Nahirniak V, Kusniak N, Kashperuk-Karpiuk I, Andrushchak L, Protsak T, Pentelejchuk N. Studying Changes of the Effective Radius in Blood Vessels after Exposure of Low Extremities to Periodical Mechanical Vibrations. $J$ Biomed Phys Eng. 2019;9(6):673-8. doi: 10.31661/jbpe.v0i0.1168. PubMed PMID: 32039098. PubMed PMCID: PMC6943842.

8. Lohman III EB, Petrofsky JS, Maloney-Hinds C, Betts-Schwab $H$, Thorpe D. The effect of whole body vibration on lower extremity skin blood flow in normal subjects. Medical Science Monitor. 2007;13(2):CR71-6. PubMed PMID: 17261985.

9. Wang L, Zhao M, Ma J, Tian S, Xiang P, Yao $W$, Fan Y. Effect of combining traction and vibration on back muscles heart rate and blood pressure. Medical Engineering \& Physics. 2014;36(11):1443-8. doi: 10.1016/j.medengphy.2014.08.008.

10.Jebur AA, Abed AY. Effect of Vibrations Transmitted Through Hand on Human Body and Blood Pressure. International Journal of Advances in Engineering Science \& Technology. 2013;3(2):47-56.

11.Robbins D, Yoganathan P, Goss-Sampson M. The influence of whole body vibration on the central and peripheral cardiovascular system. Clinical Physiology and Functional Imaging. 2014;34(5):364-9. doi: 10.1111/cpf.12103. 
Nahirniak V. M., Tsyhykalo O. V., Oliinyk I. et al

PubMed PMID: 24237890.

12.Gailiūnienè L, Krutulytė G, Šiaučiūnaitė V, Savickas $R$, Venslauskas $M$. The effect of low frequency $2-10 \mathrm{~Hz}$ vibrations on blood circulation in lower extremities. Journal of Vibroengineering. 2017;19(6):4694-701. doi: 10.21595/ jve.2017.18381.

13.Tzen Y-T, Weinheimer-Haus EM, Corbiere TF, Koh TJ. Increased skin blood flow during low intensity vibration in human participants:Analysis of control mechanisms using short-time Fourier transform. PLoS One. 2018;13(7):e0200247. doi: 10.1371/journal. pone.0200247. PubMed PMID: 30001409. PubMed PMCID: PMC6042750.

14. Herrero AJ, Menendez H, Gil L, Martin J, Martin T, Garcia-Lopez D, Gil-Agudo A, Marin PJ. Effects of whole-body vibration on blood flow and neuromuscular activity in spinal cord injury. Spinal Cord. 2014;49(4):554-9. doi: 10.1038/sc.2010.151. PubMed PMID: 21042329.

15.Folkow B, Neil E. Circulation. New York: Oxford University Press.1971. p. 593. 